\title{
Combination Therapies for Traumatic Brain Injury: Prospective Considerations
}

\author{
Susan Margulies, ${ }^{1}$ Ramona Hicks, ${ }^{2}$ and The Combination Therapies \\ for Traumatic Brain Injury Workshop Leaders*
}

Many of life's failures are people who did not realize how close they were to success when they gave up. -Thomas A. Edison (1847-1931)

\begin{abstract}
Traumatic brain injury (TBI) initiates a cascade of numerous pathophysiological events that evolve over time. Despite the complexity of TBI, research aimed at therapy development has almost exclusively focused on single therapies, all of which have failed in multicenter clinical trials. Therefore, in February 2008 the National Institute of Neurological Disorders and Stroke, with support from the National Institute of Child Health and Development, the National Heart, Lung, and Blood Institute, and the Department of Veterans Affairs, convened a workshop to discuss the opportunities and challenges of testing combination therapies for TBI. Workshop participants included clinicians and scientists from a variety of disciplines, institutions, and agencies. The objectives of the workshop were to: (1) identify the most promising combinations of therapies for TBI; (2) identify challenges of testing combination therapies in clinical and pre-clinical studies; and (3) propose research methodologies and study designs to overcome these challenges. Several promising combination therapies were discussed, but no one combination was identified as being the most promising. Rather, the general recommendation was to combine agents with complementary targets and effects (e.g., mechanisms and time-points), rather than focusing on a single target with multiple agents. In addition, it was recommended that clinical management guidelines be carefully considered when designing pre-clinical studies for therapeutic development. To overcome the challenges of testing combination therapies it was recommended that statisticians and the U.S. Food and Drug Administration be included in early discussions of experimental design. Furthermore, it was agreed that an efficient and validated screening platform for candidate therapeutics, sensitive and clinically relevant biomarkers and outcome measures, and standardization and data sharing across centers would greatly facilitate the development of successful combination therapies for TBI. Overall there was great enthusiasm for working collaboratively to act on these recommendations.
\end{abstract}

Key words: clinical trials; head injury; injury mechanisms; intervention; in-vitro and in-vivo models

\footnotetext{
${ }^{1}$ University of Pennsylvania, Philadelphia, Pennsylvania.

${ }^{2}$ National Institute of Neurological Disorders and Stroke, Bethesda, Maryland.

*Beth Ansel, National Institute of Child Health and Human Development, Bethesda, Maryland; Ross Bullock, University of Miami, Miami, Florida; David Clifford, Washington University School of Medicine, St. Louis, Missouri; Guy Clifton, University of Texas Health Science Center, Houston, Texas; Robin Conwit, National Institute of Neurological Disorders and Stroke, Bethesda, Maryland; Pramod Dash, University of Texas Health Science Center, Houston, Texas; Ramon Diaz-Arrastia, University of Texas Southwestern Medical Center, Dallas, Texas; W. Dalton Dietrich, III, University of Miami, Miami, Florida; Billy Dunn, Food and Drug Administration, Rockville, Maryland, Center for Drug Evaluation and Research, Silver Spring, Maryland; Scott Emerson, University of Washington, Seattle, Washington; Stephanie Fertig, National Institute of Neurological Disorders and Stroke, Bethesda, Maryland; Edward D. Hall, University of Kentucky, Lexington, Kentucky; David Hoyt, University of California, Irvine, California; Scott Janis, National Institute of Neurological Disorders and Stroke, Bethesda, Maryland; Walter Koroshetz, National Institute of Neurological Disorders and Stroke, Bethesda, Maryland; Daniel Laskowitz, Duke University Medical Center, Durham, North Carolina; David Meaney, University of Pennsylvania, Philadelphia, Pennsylvania; Barclay Morrison, III, Columbia University, New York, New York; Linda Noble-Haeusslein, University of California, San Francisco, San Francisco, California; John Povlishock, Virginia Commonwealth University Richmond, Virginia; Claudia Robertson, Baylor College of Medicine, Houston, Texas; Holly Soares, Pfizer Global Research and Development, Groton, Connecticut; George Sopko, National Heart, Lung and Blood Institute, Bethesda, Maryland; Pat Sullivan, University of Kentucky, Lexington, Kentucky; Michael Weinrich, National Institute of Child Health and Human Development, Bethesda, Maryland; David Wright, Emory University, Atlanta, Georgia; Ross Zafonte, Harvard Medical School, Boston, Massachusetts.
} 


\section{Introduction}

$\mathbf{T}$ RAUMATIC BRAIN INJURY (TBI) is a major medical problem (Centers for Disease Control and Prevention, 2000) for which there are management guidelines, but no Class I evidence supporting any standard therapy (McIntosh et al., 1996; Roberts et al., 1998; Cochrane, 2005; Brain Trauma Foundation, 2007). In recognition of the significant public health impact, as well as the complexity of TBI, the National Institute of Neurological Disorders and Stroke (NINDS), the National Heart, Lung, and Blood Institute (NHLBI), the National Institute of Child Health and Human Development (NICHD), and the Department of Veteran's Affairs sponsored a workshop on Combination Therapies for Traumatic Brain Injury on February 27-28, 2008. The purpose of the workshop was to convene scientists across biomedical disciplines to address the challenges and opportunities associated with selecting and testing combination therapies for TBI neuroprotection. Although the organizers acknowledged the importance of therapies aimed at regeneration and repair processes as well as neuroprotection, the scope of the workshop was limited to the first $72 \mathrm{~h}$ after TBI. The objectives of the workshop were to: (1) identify the most promising combinations of therapies based on stages and types of human TBI pathophysiology, and also on potential synergistic and antagonistic effects of the therapies; (2) identify the issues and challenges of testing combination therapies in clinical and pre-clinical studies; and (3) propose research methodologies and study designs to overcome these issues. The following is a summary of the workshop proceedings.

\section{Objective 1: Identifying Promising Combinations}

Over the past 30 years considerable research effort has been directed at understanding the secondary injury cascade that is a consequence of the primary mechanical trauma to the head. This research formed a basis for programs directed at the discovery of neuroprotective drugs for the acute treatment of TBI. As a result of these early studies, over 20 late phase II or phase III clinical trials for moderate and/or severe TBI patients were undertaken (Maas, 2007; Narayan et al., 2002). All of the clinically tested therapies failed to achieve the primary end-point of an overall benefit across the full cohort of treated patients compared to those who received the placebo treatment. The lack of success of TBI clinical trials has led scientists and clinicians to identify the probable factors for those failures, including (1) inadequate understanding of secondary injury mechanisms (e.g., translation of therapeutic windows and plasma levels between animals and humans); (2) inadequate pre-clinical testing in multiple injury models, species, ages, and genders; (3) lack of thorough investigation of pharmacokinetics; (4) a heterogeneous patient population; and (5) inadequate functional assessment scales and biomarkers for injury progression and recovery (Faden, 2002; Narayan et al., 2002; Doppenberg et al., 2004; Povlishock and Katz, 2005).

In addition to the factors cited above, the complexity of TBI is another major challenge for developing effective treatments. TBI represents a constellation of primary injury processes, which commonly include contusion, diffuse axonal injury, hematomas, and subarachnoid hemorrhage (SAH) (Adams et al., 1982; Adams et al., 1983; Moppett, 2007; Saatman et al., 2008). The initial injury typically evolves into various sec- ondary injuries such as ischemia, edema, inflammation, and brain herniation (Brain Trauma Foundation, 2007). Often multiple primary and secondary injuries coexist in TBI. However, even in cases in whom trauma appears to result in a single type of injury, numerous cellular and molecular events are initiated within minutes, hours, or days, to mediate cell damage (see Table 1 for details) (Raghupathi, 2004; Povlishock and Katz, 2005; Marklund et al., 2006; Schouten, 2007; Werner and Engelhard, 2007). In addition to the heterogeneity of the injury mechanisms, factors such as age, gender, alcohol/drug use, co-morbidities, polytrauma, and genetics can also influence the effects of an intervention following TBI (Maas et al., 2007). Some of the variability may be reduced through an improved classification system enabling the identification of people most likely to respond to the intervention (Saatman et al., 2008). In any case, the complexity of TBI provides a strong rationale for the use of combination therapies.

Another reason for exploring combination therapies for TBI is the success of this approach for other medical conditions, such as HIV/AIDS (Harrington and Carpenter, 2000; Holtgrave, 2005; May et al., 2006). After presentation of the syndrome of AIDS, HIV was rather quickly characterized, and details of the life cycle of the virus allowed rational development of drug therapy. The initial drugs developed, which inhibit the reverse transcriptase of the virus, demonstrated clear efficacy, but were frustratingly transient in their effects. The development of drugs targeted at multiple parts of the life cycle of the virus led to improvements in the prognosis for patients with HIV, and transformed a fatal disease to one that a large majority of patients can live with for decades (Mitsuyasu, 2002; Yeni et al., 2002; Cotelle, 2006; Salzwedel et al., 2007).

Currently, more than 130 single or monotherapies have demonstrated efficacy in animal models of TBI (Marklund et al., 2006). Ways to potentially increase the efficacy of seven promising single therapies by combining them with other treatments were presented at the workshop. In particular, scientific experts were asked to review what is known about the mechanisms of action of a single therapy on acute TBI pathophysiology, and then propose ways to enhance its neuroprotective and or neuroregenerative actions by using it with therapies that have complimentary, synergistic actions. Summaries of the presentations follow.

\section{Citicoline}

Citicoline (cytidine 5'-diphosphocholine or CDP-choline) is a naturally occurring endogenous compound (Kennedy et al., 2003) that may exert acute neuroprotective effects (Adibhatla and Hatcher, 2002), as well as potentiate neurorecovery in chronic TBI and stroke (Cohadon et al., 1982; Levin, 1991; Warach et al., 2000; Secades and Lorenzo, 2006). Citicoline has virtually no side effects, excellent tolerance, and welldescribed pharmacokinetics, toxicity, and bioavailability profiles (Adibhatla and Hatcher, 2005; Secades and Lorenzo, 2006). Animal data suggest that citicoline works via numerous mechanisms to attenuate neuronal injury after TBI, including increased synthesis of phosphatidylcholine, inhibition of oxidative stress and apoptotic pathways, and activation of pro-survival pathways and cholinergic and dopaminergic neurotransmission. The diversity of citicoline's mechanisms of action and pre-clinical efficiacy data make it an attractive 
Table 1. Initiation of Acute Secondary Events Post-TBI

\begin{tabular}{|c|c|c|}
\hline Within minutes ${ }^{\mathrm{a}}$ & Minutes-24 $h^{\mathrm{a}}$ & $24-72 h^{\mathrm{a}}$ \\
\hline $\begin{array}{l}\text { Cell/axon stretching, compaction } \\
\text { of neurofilaments, impaired axonal } \\
\text { transport, axonal swelling, axonal } \\
\text { disconnection } \\
\text { Disruption of the blood-brain barrier } \\
\text { Excessive neuronal activity: Glutamate release } \\
\text { Widespread changes in neurotransmitters: } \\
\text { Catecholamines, serotonin, histamine, } \\
\text { GABA, acetylcholine } \\
\text { Hemorrhage (heme, iron-mediated toxicity) } \\
\text { Seizures } \\
\text { Physiologic disturbances: Decreased cerebral } \\
\text { blood flow, hypotension, hypoxemia, } \\
\text { increased intracranial pressure, decreased } \\
\text { cerebral perfusion pressure } \\
\text { Increased free radical production } \\
\text { Disruption of calcium homeostasis } \\
\text { Mitochondrial disturbances }\end{array}$ & $\begin{array}{l}\text { Oxidative damage: Increased reactive } \\
\text { oxygen and nitrogen species } \\
\text { (lipid peroxidation, protein oxidation, } \\
\text { peroxynitrite), reduction in endogenous } \\
\text { antioxidants (e.g., glutathione) } \\
\text { Ischemia } \\
\text { Edema: Cytotoxic, vasogenic } \\
\text { Enzymatic activation: kallikrein-kinins, calpains, } \\
\text { caspases, endonucleases, metalloproteinases } \\
\text { Decreased ATP: Changes in brain } \\
\text { metabolism (altered glucose utilization } \\
\text { and switch to alternative fuels), } \\
\text { elevated lactate } \\
\text { Cytoskeleton changes in cell somas and axons } \\
\text { Widespread changes in gene expression: cell } \\
\text { cycle, metabolism, inflammation, receptors, } \\
\text { channels and transporters, signal transduction, } \\
\text { cytoskeleton, membrane proteins, } \\
\text { neuropeptides, growth factors, and proteins } \\
\text { involved in transcription/translation } \\
\text { Inflammation: Cytokines, chemokines, cell } \\
\text { adhesion molecules, influx of leukocytes, } \\
\text { activation of resident macrophages }\end{array}$ & $\begin{array}{l}\text { Non-ischemic } \\
\text { metabolic failure }\end{array}$ \\
\hline
\end{tabular}

Traumatic brain injury rapidly initiates a series of secondary events that collectively contribute to cell injury and/or repair. These secondary events often create long-term neurological consequences, including cognitive dysfunction. Based primarily upon rodent models of TBI, these early events can generally be divided into three periods, beginning with those that arise within minutes after injury, to those that evolve over the first $24 \mathrm{~h}$, and finally to events that may be more delayed in onset, appearing between 24 and $72 \mathrm{~h}$ post-injury.

For further details see Clark et al., 1997; Floyd, 1999; Graham et al., 2000; Graham et al., 2002; Lo et al., 2002; Smith et al., 2003; Raghupathi, 2004; Unterberg et al., 2004; Povlishock and Katz, 2005; Sofroniew, 2005; Thompson et al., 2005; Marklund et al., 2006; Yi and Hazell, 2006; Floyd and Lyeth, 2007; Morganti-Kossmann et al., 2007; Pasternak and Lanier, 2007; Schouten, 2007; Werner and Engelhard, 2007.

${ }^{a}$ Each of these periods reflects only an estimation of the onset of these pathogenic events, as details about their temporal profile and interactions are incompletely understood, but most extend for days post-TBI. Variability in onset and frequency, as well as the duration of these events, is governed in part by the type and magnitude of the injury.

candidate for therapeutic development, and a large multicenter trial for TBI is currently underway. Citicoline has already been used for pre-clinical stroke studies in combination with tPA, urokinase, or MK-801(Hickenbottom and Grotta, 1998). For TBI, citicoline should be combined with treatments that complement its actions on neuronal injury, such as drugs that target axonal injury or have anti-inflammatory actions. Promising potential agents to combine with citicoline include hypertonic saline, statins, progesterone, erythropoietin, and cyclosporine A. Detailed investigations regarding route of administration and brain uptake are needed, as well as mechanistic studies to evaluate the effect of a second treatment on citicoline's therapeutic effects.

\section{Erythropoietin}

Erythropoietin (Epo) is a hematopoietic growth factor that is produced in the kidney and in fetal liver. Binding to the Epo receptor (EpoR) controls the terminal maturation of red blood cells (RBCs), stimulating production of RBCs. Epo is approved for clinical use in the treatment of chronic anemia associated with kidney disease, cancer, and HIV. There is increasing interest in the use of Epo in critically ill patients to reduce the need for blood transfusion and its related problems (Corwin et al., 1999; Corwin et al., 2002). A recent large clinical trial suggested that Epo may also reduce mortality in trauma patients (Corwin et al., 2007). EpoR is expressed in the brain and Epo is produced in the brain in response to injury (Digicaylioglu et al., 1995; Juul et al., 1999). Epo administration is neuroprotective in a wide variety of CNS injury models, including trauma. Epo is an attractive candidate for treatment of TBI because it is neuroprotective and enhances recovery, has few side effects, and has a practical therapeutic time window of $6 \mathrm{~h}$ (Brines et al., 2000; Cherian et al., 2007). When Epo is given early post-injury, the following neuroprotective mechanisms may play a role: Epo inhibits apoptosis, Epo has antiinflammatory activity, and Epo reduces cerebral vasospasm and improves cerebral blood flow (CBF) (Brines et al., 2000; Grasso, 2001; Springborg et al., 2002; Chong et al., 2003a; Chong et al., 2003b; Kanagy et al., 2003). Even with delayed Epo administration, the following effects may still be beneficial: Epo enhances neurogenesis and angiogenesis (Marti et al., 2000; Springborg et al., 2002; Wang et al., 2004; Lu et al., 2005), and Epo increases the hematocrit, reducing the need for blood transfusion. The only serious side effect of Epo for the TBI patient population is that Epo increases the risk of deep vein thrombosis (DVT) by twofold (Corwin et al., 2007). However, a subgroup analysis of a large clinical trial revealed that prophylactic heparin administration prevents the increased risk of DVT caused by Epo administration in critically ill patients (Corwin et al., 2007). In developing combination treatments, previous studies have examined potential synergistic effects with Epo. Specifically, investigators found that Epo augments the increase in CBF that is induced 
by L-arginine in contused brain after cortical impact injury (Cherian et al., 2007). Second, steroid administration after experimental spinal cord injury reduces the antiinflammatory effect of Epo (Gorio et al., 2005).

\section{Hypothermia}

Hypothermia activates cell survival pathways via ERK signaling (Atkins et al., 2007), and attenuates many of the neurological sequelae of TBI, including altered brain metabolism (Vink et al., 1987; Kaibara et al., 1999), tissue loss (Bramlett et al., 1997), axonal damage (Koizumi and Povlishock, 1998), microvascular dysfunction (Smith and Hall, 1996; Suehiro et al., 2003), edema (Mansfield et al., 1996; Sahuquillo and Vilalta, 2007), and behavioral abnormalities (Clifton et al., 1991; Bramlett et al., 1997). These positive effects generated enthusiasm and support for two Phase III trials of hypothermia for TBI, one for adult and one for pediatric populations. However, important questions remain regarding the optimal temperature, treatment period, and therapeutic window for maximizing neuroprotection and minimizing systemic complications (Brain Trauma Foundation, 2007; Peterson et al., 2008). Recent evidence also suggests that hypothermia constitutes a beneficial platform for the subsequent use of additional therapies. For example, post-traumatic hypothermia significantly extended the therapeutic window of oxygen radical scavengers (Baranova et al., 2008), and their combined use also had additive effects on vascular protection. While the mechanisms are unclear, one possibility is that hypothermia provided this enhanced protective effect via its reduction of brain metabolism and drug degradation/clearance (Tortorici et al., 2007), However, while evidence suggests that hypothermia may extend the therapeutic window of other interventions, it may also slow the pharmacokinetic and pharmacodynamic properties of them as well.

\section{Progesterone}

Progesterone, while best known for its effects on the female reproductive system, also has abundant receptors in the CNS of males and females (Camacho-Arroyo et al., 1994; CamachoArroyo et al., 1996; Genazzani et al., 1996), and has long been known to influence neuronal differentiation during fetal development. Progesterone is a pleiotropic drug that has been shown to (1) protect and reconstitute the blood-brain barrier, (2) reduce cerebral edema through decreasing vasogenic and cytotoxic edema and modulating brain water regulation via aquaporin channels, (3) downregulate the inflammatory cascade and pro-inflammatory cytokines in response to neurotrauma, (4) reduce free radicals and lipid peroxidation, and (5) decrease apoptosis (Camacho-Arroyo et al., 1994; Camacho-Arroyo et al., 1996; Genazzani et al., 1996; Limmroth et al., 1996; Reddy and Kulkarni, 1996; Baulieu and Schumacher, 1997; Wright et al., 2001; Stein et al., 2007). Two Phase II trials have demonstrated its safety and potential benefits (Wright et al., 2007; Xiao et al., 2008), and Phase III trials are now in preparation. To further enhance the pleiotropic effects of progesterone on TBI, it might be advantageous to combine it with agents that (1) protect the intracerebral vasculature, (2) diminish the effects of glutamate release and calcium influx, (3) more directly protect the mitochondria, (4) protect against the toxic effects of heme breakdown products, (5) enhance free radical scavenging, (6) enhance cerebral blood flow, (7) modulate the kallikrein-kinin system, (8) protect the axonal and cytoskeleton infrastructure, and (9) protect against diffuse axonal injury. Additionally, combining progesterone with hypothermia may be beneficial, especially during the rewarming stage of therapy.

\section{Cyclosporine $A$}

Cyclosporine A (CsA) attenuates mitochondrial failure, which is known to be an important injury mechanism in TBI. Mitochondrial failure leads to energy imbalance, ionic imbalance, swelling of mitochondria, pro-apoptotic events, reduced brain ATP levels, and release of cytochrome C (Sullivan et al., 2005). The locus of action for CsA is in stabilizing the mitochondrial transition pore (Sullivan et al., 2000). Several pre-clinical TBI and ischemia studies (mostly in rodents) have demonstrated neuroprotection (Folbergrova et al., 1997; Li et al., 2000; Sullivan et al., 2000; Alessandri et al., 2002; Ferrand-Drake et al., 2003; Fukui et al., 2003; Hansson et al., 2003; Suehiro et al., 2003; Signoretti et al., 2004). The advantages of CsA are that it is FDA-approved and off-patent, and therefore is inexpensively manufactured by several companies, and it has well-described safety and dosing profiles. CsA is also one of the most potent stabilizers of the mitochondrial transition pore. Secondary to the inhibition of mitochondrial transition pore opening, CsA also attenuates mitochondrial free radical oxidative damage to mitochondrial proteins and thus it acts as an indirect antioxidant (Mbye et al., 2009). A disadvantage is that chronic usage adversely impacts the immune system, but acute usage for TBI neuroprotection satisfied a broad range of safety parameters in a Phase I clinical trial. Another disadvantage is that CsA has relatively poor brain penetration; however, improvements in increased cerebral perfusion pressure, improved glucose levels, and reduced brain swelling were noted in the Phase I trial (personal communication, R. Bullock). Efforts to block excretion of CsA from the brain with ketoconazole were not successful. Phase III trials for CsA are now in preparation. Because of its slow entry into the brain $(6 \mathrm{~h})$, the mitochondrial benefits of CsA might be enhanced if combined with hypothermia, by both prolonging the treatment window and through their synergistic effects on preservation of brain bioenergetics, but combining CsA with hypothermia has the potential risk of infection because of the immune suppression. Because of this potential risk, one might exclude patients with multiple injuries from combined treatment with CsA and hypothermia. CsA might also be used in combination with NMDA inhibitors to block calcium flux, a precipitator for the mitochondrial damage, thus enhancing mitochondrial protection. One pharmacological caveat is that the neuroprotective doseresponse curve for CsA is biphasic, so using it in combination would require a very careful evaluation of the pharmacokinetics and dose response.

\section{Statins}

Statins are 3-hydroxy-3-methylglutaryl coenzyme A (HMGCoA) reductase inhibitors, which are potent inhibitors of cholesterol biosynthesis. There is growing clinical and preclinical evidence that the statin class of drugs may have additional pleiotropic properties that are potentially neuroprotective, independent of their effect on serum cholesterol (Cucchiara and Kasner, 2001). For example, in the acute phase 
of TBI, statins exert anti-inflammatory effects (Weitz-Schmidt et al., 2001; Chen et al., 2007; Wang et al., 2007), which may reduce the later development of cerebral edema and intracranial hypertension. Statins also cause an upregulation of eNOS and stabilize endothelial surfaces (Laufs et al., 1998), which may result in improved cerebral perfusion following trauma (Lu et al., 2005; Wang et al., 2007). In the subacute period and chronic period following cerebral injury, statins may facilitate recovery via their effects on neurogenesis and angiogenesis (Chen et al., 2003; Lu et al., 2004a; Lu et al., 2007). In addition to these multiple mechanisms of action, there are a number of features that make the use of statins attractive in the treatment of acute brain injury. Based on pre-clinical observations in a murine model of SAH (McGirt et al., 2002), statins have been demonstrated to reduce clinical and radiographic vasospasm and improve outcome following aneurysmal SAH (Lynch et al., 2005; Tseng et al., 2005). Pre-clinical evidence also suggests that the use of statins improve outcomes in rodent models of TBI (Lu et al., 2004; Lu et al., 2004a $\&$ b) and intracranial hemorrhage (Jung et al., 2004; Lu et al., 2004c; Seyfried et al., 2004). Statins are well tolerated, easy to administer, and have a long, safe clinical track record. Adverse events, primarily myopathy and transaminitis, have been well defined and can be easily monitored. Moreover, clinical experience suggests that statins are well-tolerated in patients with life-threatening neurological disease (Lynch et al., 2005; Tseng et al., 2005). Thus statins may represent a novel adjunct strategy in combination therapy treatments for TBI.

\section{Hypertonic saline}

Hypertonic saline is an attractive treatment for TBI because it restores blood pressure, increases organ blood flow, exerts a positive inotropic effect, and is thought to mobilize water across the intact blood-brain barrier by dehydrating endothelial cells and erythrocytes (Tommasino and Picozzi, 2007). It also affects leukocyte adhesion and reduces the inflammatory response to injury (Pascual et al., 2003). The effects of hypertonic saline on TBI include improved hemodynamics through plasma volume expansion, vasoregulation via effects on vascular endothelium, a decrease in cerebral edema, and cellular modulation through both immunologic and excitotoxic effects (Doyle et al., 2001). Hypertonic saline has been used extensively in the pre-hospital arena and in ICUs around the world. There have been several clinical trials evaluating hypertonic saline in TBI. The safety profile is good and improvements in intracranial pressure and survival have been observed, but no improvements have been seen in functional outcomes. Currently a large randomized clinical trial of pre-hospital treatment with hypertonic saline versus normal saline in patients with TBI is in progress. Hypertonic saline should be strongly considered for use in conjunction with other promising therapies that target neuronal and axonal injury mechanisms.

\section{Objective 2: Challenges for Testing Combination Therapies}

Pharmacokinetics, pharmacodynamics, regulatory considerations, and experimental design and analysis were identified as important topics for discussion during the workshop. Scientific experts were invited to give presentations to launch the discussions, which are summarized below.

\section{Pharmacokinetics and pharmacodynamics}

Pharmacokinetics and pharmacodynamics may be altered, not only when two drugs are combined, but also when a drug is combined with another treatment. Thus it is critically important to study these interactions in parallel with the evaluation of the therapeutic effect. Specifically, a careful dose-response analysis needs to be performed for the combination treatment. Ideally, the combination would not only allow for a greater level of protective efficacy (improved pharmacodynamics), but also allow for lower doses of either drug to be administered, such that the therapeutic index (i.e., safety margin) is also improved over that seen with either drug alone. The combined administration of two or more drugs could also result in altered pharmacokinetic interactions with regard to absorption (in the case of orally administered drugs), distribution (e.g., plasma protein binding and brain penetration), metabolism (mainly hepatic), or renal excretion. Similarly, hypothermia could significantly modify the pharmacokinetics of a drug by modifying cardiac output and organ blood flow or hepatic metabolic rate.

An example of a combination therapy for enhanced neuroprotection that could potentially alter the pharmacokinetics is the co-administration of tirilazad (the 21-aminosteroid lipid peroxidation inhibitor) (Hall et al., 1994), and the mitochondrial protective agent CsA. Both drugs have less-than-ideal brain penetration because they are known to interact with the blood-brain barrier P-glycoprotein that pumps them out of the brain. However, when administered together, a secondary benefit might be that they enhance each other's brain penetration, since they would compete for the P-glycoprotein efflux mechanism. Because there are many such possible interactions, pre-clinical pharmacokinetic studies are needed in order to maximize both efficacy and safety in subsequent clinical trials of combination therapies for TBI.

\section{Regulatory considerations}

Regulatory considerations for combination therapies are the responsibility of the FDA. There are three main centers that deal with human clinical research: the Center for Drug Evaluation and Research (CDER), the Center for Biologics Evaluation and Research (CBER), and the Center for Devices and Radiological Health (CDRH). Products that fall under the purview of these centers may be classified as a combination product, as explicitly defined in 21 CFR 3.2(e) (U.S. Food and Drug Administration, 2008). Operationally, such a product exists if two or more components are combined into a single entity, two or more components are packaged together, an investigational component is used with an approved product but packaged separately with cross-labeling, or separate investigational components are packaged separately with cross-labeling. Drug-drug combinations are not defined as combination products by the FDA and represent a distinct regulatory entity. Such combinations are referred to as fixedcombination drugs and are defined in 21 CFR 300.50 (U.S. Food and Drug Administration, 2008). This regulation states, in part, that two or more drugs may be combined in a single dosage form when each component makes a contribution to the claimed effects. This has implications for study design during clinical development. In a typical situation, it is not sufficient to simply study the combination versus placebo, nor is it sufficient to study the combination versus only one of the 
two components. A full factorial design is typically used to demonstrate the benefit of fixed-combination drugs. Aside from the requirement to demonstrate that each component of a fixed-combination drug makes a contribution to the claimed effects, the approval process for fixed-combination drugs is normally similar to that of individual drugs.

Combination products contain components that are subject to the regulatory requirements of more than one center. The Office of Combination Products (OCP) at the FDA has broad responsibilities covering the regulatory life-cycle of combination products. The formal duties of the OCP are found in 21 USC 353(g) (U.S. Food and Drug Administration, 2008). For the purposes of this discussion, one of the more important duties is assigning CDER, CBER, or CDRH primary jurisdiction for review of a combination product., It is important to note that primary regulatory responsibilities for, and oversight of, specific combination products will remain in the assigned center, not the OCP. Assignment of primary jurisdiction is based on a determination of the primary mode of action (PMOA), defined as the single mode of action (MOA) of a combination product that provides its most important therapeutic action. MOA is the means by which a product achieves its intended therapeutic effect or action. If the PMOA is indeterminate, such as when a product has independent MOAs, neither of which is subordinate to the other, an algorithm is used for assignment. This algorithm first examines the new product's consistency with other combination products raising similar safety and effectiveness questions, and assigns the new product to the center that had jurisdiction over these similar products. If no similar products are suitable for comparison, then the new product is assigned to the center with the greatest expertise to evaluate the most significant safety and effectiveness questions raised by the combination product. The center of primary jurisdiction is of significant importance to both the sponsor and the FDA because although all centers are charged with determining safety and effectiveness, the specific regulations governing this determination may differ. Even once a primary center has been identified, however, the additional centers may remain involved in the review process in a consultative or collaborative role.

In summary, combination therapies represent a distinct regulatory entity and a sponsor may face unique challenges in their clinical development. Familiarity with the issues involved in the review of a combination therapy will enhance the opportunity for a successful outcome. Both the various FDA centers and the OCP are appropriate resources for investigators contemplating a combination product submission. Investigators contemplating a submission for a combination product or fixed-combination drugs were encouraged to consult the FDA early in the experimental design process.

\section{Study design and statistical considerations}

Study design and statistical considerations are also critical for reaching the ultimate objective of randomized clinical trials for combination therapies, which is to demonstrate a large synergistic effect between treatments A and B. Whereas the objective of single-therapy trials is to prove that treatment A is clinically better than the current standard of therapy, the combination therapy trial needs to show that the combination of treatment $\mathrm{A}$ and $\mathrm{B}$ is clinically better than treatment $\mathrm{A}$ alone, treatment $\mathrm{B}$ alone, and better than the current standard therapy. In general, combination therapy studies should use a factorial experimental design with one-fourth of the subjects receiving treatment $A$, one-fourth receiving treatment $B$, onefourth getting neither A nor B, and one-fourth receiving $A$ and $\mathrm{B}$ together. A complete analysis of the groups would involve all six possible comparisons of the four groups. However, if the single therapies by themselves are not effective, then the standard analysis could eliminate the comparisons of treatment $\mathrm{A}$ to non-treatment $\mathrm{A}$, and treatment $\mathrm{B}$ to non-treatment $\mathrm{B}$, and only compare the combination treatment $\mathrm{A}+\mathrm{B}$ to the other three groups. Alternatively, if the single therapies are effective, then one should conduct a complete factorial analysis.

When combination therapies are administered sequentially, investigators might consider an experimental design in which patients are randomized to the first therapy, and then those patients who survive to the next phase are rerandomized for the second intervention. This design provides the most statistical power, with equal numbers in the treatment arms. However, sequential combination studies also increase the risk for missing data, because not all subjects will make it to the second treatment. This needs to be addressed and the end-points are defined in a way that covers all eventualities. Another suggestion for more efficient testing of sequential combination therapies is to incorporate interim analyses into the study design to determine the efficacy of the first treatment alone. The exclusion criteria required for combination therapies raises another challenge. The exclusion criteria for the study population should include anyone who has contraindications for either treatment $\mathrm{A}$ or B. For sequential combination treatments one must consider not only contraindications at baseline, but must anticipate that one of the treatments might lead to adverse effects that appear as a contraindication to the second treatment. As with all randomized controlled trials, one encounters barriers due to safety concerns for treatment A or B, as well as fear of attenuated effects. Another concern is the increased potential for bias related to safety profiles. For example, if one treatment leads to more or less hemorrhaging on CTs and that increases surveillance, then that situation may bias ascertainment of surrogate or clinical outcomes as the trial continues. Therefore, combination therapy trials may end up focusing on a highly specific patient population, which may create challenges for enrolling the required number of patients.

Similar to studies of single therapies, the relevant outcome metrics for monitoring combination therapy efficacy fall into two major categories: surrogate outcomes, and functional outcomes. Surrogate outcomes are useful in pre-clinical and Phase I and II clinical studies to reveal treatment actions and interactions, and to determine the relative advantage of concurrent versus sequential administration on the time course of TBI. Functional outcomes are the best metrics to evaluate efficacy, effectiveness, and safety.

In addition, interim analyses of futility (also referred to as non-inferiority studies) should be considered in both monoand multi-therapy clinical trials, as they substantially lower sample size and have the potential to reject drugs or treatment designs which are clearly ineffective. A futility experimental design incorporates the view that in the early phases of clinical development of a new therapy, it is in fact riskier to discard a potentially useful treatment than it is to fail to 
definitively identify efficacy. In contrast, in traditional studies, it is customary to set alpha at 0.05 (the likelihood of a falsepositive result of less than $5 \%$ ), and beta at 0.2 (the likelihood of a false-negative result-or that a beneficial effect will be missed-is less than $20 \%$ ). However, in a futility study the null hypothesis is that treatment has promise and will therefore produce results exceeding a meaningful threshold. Thus, an alpha of 0.1 in a futility study means that the chance of beneficial effect being missed is less than $10 \%$. In a futility design, if the efficacy threshold is not met, the null hypothesis is rejected and further study of the treatment is considered futile (Schwid and Cutter, 2006). Futility design trials were pioneered in cancer chemotherapy studies, and have recently been used in Phase II clinical trials of neurological disorders such as Parkinson's disease (Tilley et al., 2006) and stroke (Palesch et al., 2005).

\section{Objective 3: Optimized Strategies for Developing Single and Combination Therapies}

As part of the workshop, three parallel breakout sessions discussed the steps required to develop combination therapies for TBI using in-vitro and in-vivo pre-clinical models, and clinical trial platforms. During the discussions it became apparent that many of the strategies put forward were also relevant to the development of single therapies for TBI.

\section{In-vitro TBI models}

In-vitro TBI models have higher throughput than in-vivo models. They offer lower-cost alternatives for evaluating a wide range of timing and dosing parameters, which increase geometrically when testing combination therapies. In-vitro models aim to reproduce the traumatic tissue distortion of TBI to activate some of the same secondary injury mechanisms that occur in vivo (animals and humans). However, like all model systems, they have limitations, which need to be considered carefully when developing a screening platform for TBI.

The first consideration is which in-vitro model or models to use. Substrate deformation models reproduce non-penetrating injuries such as falls, blunt impacts, and inertial loading, and are advantageous because of ease of use and commercial availability. One caveat is that not all systems produce uniform deformations. Transection models scratch or cut cultured cells or brain slices, are easy to use, and most closely mimic penetrating head injuries (Tecoma et al., 1989; Mukhin et al., 1997; Toni et al., 1997; Mukhin et al., 1998). Crush or weight drop models use an indenter to press on cultured tissue slices and reproduce contusion-type injuries (Sieg et al., 1999; Frantseva et al., 2002; Bendel et al., 2004). Fluid shear models induce cellular deformation with rotating fluid flow between two plates and are advantageous in that cells can be imaged during application of the fluid shear (LaPlaca and Thibault, 1997; Edwards et al., 2001; Serbest et al., 2005). In hydrostatic pressure models, cells or tissues are placed in a chamber which is pressurized, either with a transient pressure pulse (similar to a fluid percussion injury) or a tonic applied load (Panizzon et al., 1998; Etzion and Grossman, 2000; Panickar et al., 2002). Combined injury models have also been developed. For example, substrate deformation models and transection models have been combined with an ischemia/hypoxic or NMDA excitotoxic challenge, and the combination model demon- strated greater necrotic and apoptotic cell death than each injury model alone (Cargill and Thibault, 1996; Allen et al., 1999; Glass et al., 2002; Arundine et al., 2004; Glass et al., 2004; Engel et al., 2005). Rather than developing additional models, the in-vitro break-out group recommended that there be greater commercialization and standardization of existing models, and hands-on training programs to learn how to use them.

A second consideration is the choice of a cell or tissue culture system to best represent the in-vivo environment. Cell lines (e.g., PC12, NT2, SH-SY5Y, and NG-108 cells) are generally easier to use than primary cell culture, and can be differentiated from their mitotic state into a neuronal-like phenotype. These cell lines can also be manipulated at the molecular level to facilitate broad screening for new drug discovery in single and combination therapies for TBI. Dissociated primary cell cultures (neurons, astrocytes, oligodendrocytes, microglia, and mixed cultures) from embryonic or neonatal brains are the most widely used culture system, and data suggest that mixed cultures respond differently than homogeneous cultures (Mukhin et al., 1997; Katano et al., 1999; Ahmed et al., 2000; Lea et al., 2002; Lea et al., 2003). The effects of the physical manipulations during harvest and suspension are unknown, but receptor expression profiles likely vary as the cells mature over days and weeks in culture. Caution should be used when comparing across culture conditions and extrapolating results to the adult. Immature organotypic brain slice cultures (200-400 $\mu$ m thick) from cortex (Elkin and Morrison, 2007), hippocampus (Stoppini et al., 1993; Hong and Chang, 1995), and thalamus (Sieg et al., 1999) have been maintained in culture for extended periods of time. A significant advantage of slice cultures is that the in-vivo cellular interconnections and heterogeneity are maintained in these cultures. Drawbacks include a lack of vasculature, some remodeling of intrinsic circuitry (Gahwiler, 1984; Zimmer and Gahwiler, 1984; Caeser and Aertsen, 1991; Robain et al., 1994; Kamada et al., 2004), and maturation during culture (Buchs et al., 1993; Bahr et al., 1995; Collin et al., 1997; Hartel and Matus, 1997). An advantage over dissociated cultures, the slice may be able to clear dead cells and severed processes within the first 5 days in culture (De Simoni et al., 2003), fill denuded dendritic fields, and re-establish connections. For all in-vitro model systems, standardization of methods across laboratories would improve inter-laboratory consistency in therapeutic development.

A third consideration is what outcomes to measure to capture clinically relevant information. There are many options, including measures of neuronal and astrocytic death, assessment of biochemical or electrophysiological function, alterations in gross and microscopic structures, and released biomarkers that could be used to "synchronize" time-lines between in-vitro, in-vivo, and human injury responses. Interestingly, swelling and bulbs in axons or the morphological changes seen in the injured cortex and hippocampus in vivo (Adams et al., 1982; Adams et al., 1983; Povlishock et al., 1983; Povlishock, 1992; Foda and Marmarou, 1994) are only rarely reported for in-vitro models (Gross et al., 1983; Emery et al., 1987; Gross and Higgins, 1987; Lucas et al., 1990; Stoppini et al., 1993; Smith et al., 1999; Morrison et al., 2003).

In summary, further research to establish the validity and fidelity of in-vitro models is recommended. Once this is established, it is expected that in-vitro models could be used 
to test permutations and combinations of therapies more rapidly than the in-vivo models. Furthermore in-vitro models could be used to scan for new compounds with available small molecule libraries, and provide a valuable new dimension for developing therapies. Given the heterogeneity of human TBI, it is important to select the relevant in-vitro model and cell/tissue when testing the effectiveness of therapies.

\section{In-vivo TBI models}

In-vivo TBI models play a key role in optimizing the design of clinical trials. Criteria for success have been established by both the Stroke Therapy Academic Industry Roundtable (STAIR) working group (STAIR, 1999; STAIR-II, 2001; Feuerstein et al., 2008; Fisher et al., 2009), and by a TBI focus group (Narayan et al., 2002). These criteria are relevant to development of both single and combination therapies for TBI and are summarized in Table 2. However, in addition to meeting these study design criteria, combination therapies should be designed to achieve at least one of the following goals: (1) affect multiple targets; (2) produce synergistic effects on a single target (e.g., by convergent signaling pathways), (3) increase distribution and half-life or decrease toxicity, (4) target sequential stages of injury (e.g., combining acute neuroprotection with agents that promote brain repair), (5) involve different domains (such as drugs, delivery devices, or even activity-based rehabilitative strategies), or (6) focus on either novel therapeutics or previously tested drugs in combinations.

The end-points for combination trials in animal models should include early measures of drug activity to identify potential negative interactions on treatment effect, metabolism, absorption, toxicity, or brain penetration. In addition,

Table 2. Study Design Considerations for Pre-Clinical TBI Single and Combination Therapy Development ${ }^{a}$

- Was the candidate therapy evaluated in multiple models and in both rodent and gyrencephalic species?

- Was the dose-response effect evaluated over a clinically relevant time window?

- Did the studies include clinically relevant physiological monitoring?

- Were the studies blinded and randomized?

- Were the pharmacokinetics assessed in the target tissue of the experimental species and under conditions that reflect real-life clinical situations?

- Were surrogate markers evaluated to determine if the therapy attenuates the specific injury mechanisms that are being targeted?

- Were histological and functional outcome measures assessed following a prolonged survival interval to ensure that early treatment effects are not diminished?

- Was the candidate therapy evaluated in both genders, across the life-span, and across the spectrum of injury severities?

- Were the treatment effects replicated in several laboratories and/or observed in a multicenter pre-clinical consortium?

a Based on the STAIR Recommendations for Preclinical Stroke Drug Development (STAIR, 1999; STAIR, 2001; Fisher et al., 2009), a workshop on clinical trials in head injury (Narayan et al., 2002), and the current workshop. because of the importance of co-morbidities and physiological responses in the severely head-injured population, special care should be taken to measure interactive effects on clinically important parameters such as vulnerability to infection, hemodynamic variables, temperature, edema, and electrolyte homeostasis. Pre-clinical toxicology studies of the combined agents may be necessary to ensure safety prior to entering a clinical trial. Untoward interactions are a major concern, even with previously approved drugs whose activity or doseresponse may be altered in the presence of another active agent. Retesting of dose-response curves is especially important for agents with a narrow therapeutic index or a biphasic dose-response curve.

Ideally, many combination therapies should undergo preclinical testing, with the best combinations chosen for further clinical testing. To prioritize those combinations it is important to develop standardized screening experiments in animal models so that results from different combinations can be compared across laboratories and species. Both positive and negative data from the animal experiments should be made publicly available so that research may focus on the most promising combinations, and unnecessary redundancy is avoided. For an initial proof of principle pre-clinical study, the combination therapy group might be investigated before inclusion of single-therapy groups. The improvements could be in efficacy or in therapeutic safety. Prioritizing treatments that have demonstrated efficacy as a single therapy (e.g., CsA and hypothermia) may be the most efficient strategy for initial screening. Later, direct pre-clinical comparisons of promising combinations could help prioritize them for future clinical testing. A comprehensive pre-clinical study design must also optimize a dosing methodology (dose, route, and timing), evaluate the mechanism of action, and assess the pharmacokinetic properties of the combination treatment. Definitions of failure include demonstrating loss or neutralization of activity of either component of the combination, increased mortality, or failure to improve the effect of either single therapy. The eventual study design should parallel clinical studies in terms of treatment groups, delay to treatment, dosing, and the time course of the evaluation of responses. Finally, standards of care in pre-clinical studies should be designed to include standards of care routinely followed in clinical trials (e.g., blood pressure and management of intracranial pressure).

In summary, pre-clinical studies play an essential role in combination therapy development for TBI. To ensure optimal translation to Phase I and II clinical trials, pre-clinical studies of combination therapies should include testing in multiple animal models, characterization of trauma-response relationships and pharmacokinetics, and utilization of multiple surrogate markers of efficacy and multiple functional outcome measures relevant to the clinical population (Table 2). The creation of a consortium of laboratories for conducting coordinated pre-clinical screening and efficacy studies, with uniform standards of animal care and outcome measures, could contribute to the development of both single and combination therapies for TBI.

\section{Clinical trials}

Clinical trials for combination therapies will require efficient in-vitro and in-vivo pre-clinical efficacy studies as previously discussed to select the best combinations among the 
many possibilities. Also, it should be noted that drugs previously tested and shown to be ineffective as a single therapy should not necessarily be excluded as possible candidates for use in combination therapy. This is based in part on the caffeinol study, in which ethanol plus caffeine produced a dramatic reduction in overall infarct volume in a model of ischemic stroke, although neither therapy worked as a single therapy (Strong et al., 2000; Aronowski et al., 2003). Drugs that have multiple and pleiotropic mechanisms of action and target multiple mechanisms may be advantageous for TBI because of its complexity and heterogeneity (Table 1). Also, TBI often occurs along with multiple types of injuries to other organs (polytrauma). Thus, combining two drugs with multiple mechanisms of action may produce the largest effects for such a heterogeneous injury. Alternatively, one could select therapies that exert neuroprotective actions on a single specific target, but this approach may be premature until a new classification system to reduce the heterogeneity of subjects is available (Saatman et al., 2008), and until more TBI therapies have a well-understood mechanism of action.

The temporal evolution of TBI is another important issue, especially when designing effective serial combination therapies. The focus of this NIH workshop was on the first $72 \mathrm{~h}$ after injury (Table 1 ) because early intervention has the potential to prevent secondary injuries, and most of the data on mechanisms of TBI injury are on acute injury. Consequently, it is important to consider agents that act over clinically relevant time periods and can be given via practical routes of administration. For example, during the first $30-60 \mathrm{~min}$ post-injury, when the patient is typically cared for by paramedics during transport to the hospital, the problems receiving the most attention are maintenance of adequate ventilation, blood pressure, and pulse. A comprehensive sequential combination treatment for acute TBI might be early administration of chilled hypertonic saline plus L-arginine (Prough et al., 2006) to provide a small-volume resuscitation, followed by Epo at the emergency medical center. Another rapid response treatment that could be applied in the field is hyperoxia.

Combination therapies also increase the need for widely accepted, validated biomarkers (clinical, biochemical, or imaging) to detect and monitor secondary injuries. Ongoing studies using novel MRI methods and proteomic strategies hold much promise, but substantial work remains to be done. Identification of these biomarkers will greatly facilitate development of therapies, and will be of particular use in combination therapies for understanding the interactions. For example, if combining drugs produces negative or synergistic effects, biomarkers will help to determine the reason for these non-additive effects.

Detecting additive or synergistic effects of therapies used in combination, a requirement of the FDA, will require more sensitive and specific outcome measures. The commonly used dichotomized Glasgow Outcome Score (GOS) and Glasgow Outcome Score-Extended (GOS-E) are insensitive to the important but subtle deficits in memory, executive function, and affect that produce significant disability (McCauley et al., 2001). Furthermore, because disability after TBI encompasses multiple domains of dysfunction, a single functional assessment scale may not be able to identify important deficits in all patients. There are several mathematical approaches to compare two groups with respect to more than one outcome. The options available include using Bonferroni or other adjust- ments for multiple comparisons, reducing the dimensions of the problem by averaging the outcomes, or applying a global test based on multiple correlated binary outcomes (Lefkopoulou and Ryan, 1993; Lu and Tilley, 2001). Of these, the latter approach has been found to be useful in a variety of clinical settings. Incorporating several different measures, which although correlated measure different domains of dysfunction after TBI, significantly lowers the sample size required. The recently completed magnesium sulfate clinical trial for TBI used a composite outcome measure (Temkin et al., 2007). Center-to-center variability becomes even more important when using combination therapies, because this by itself increases the complexity of the study protocol and monitoring. Plus, anything that increases "noise" in the data will have a negative effect on detecting additive effects of the combination therapies that are required for the pilot studies.

In summary, attractive candidates for combination therapies should be based not only on robust pre-clinical efficacy data, but also address the temporal evolution and heterogeneous phenotype of TBI. Thus, using therapies that target multiple mechanisms rather than a single mechanism is recommended. Using composite outcome measures and standardization of patient care protocols across trial centers may be useful for minimizing the required number of subjects and for detecting significant effects.

\section{Summary of Recommendations}

The workshop participants (listed below) agreed that the heterogeneity of TBI provides a strong rationale for the hypothesis that combination therapies will improve clinical outcomes compared to current single-agent interventions. Several steps were identified for moving research on combination therapies forward, including the following:

- Select therapies for use in combination that target multiple and complementary mechanisms of action.

- Validate surrogate markers to monitor treatment effects on brain injury and recovery for all stages of therapy development (in-vitro, animal, and human).

- Develop in-vitro, animal, and clinical platforms for coordinated studies across multiple laboratories.

- Use efficient designs for trials and data analysis.

- Be informed of the FDA regulations.

- Adopt a uniform standard of care for clinical trials, and mimic these standards in pre-clinical studies.

- Establish a shared database of positive and negative clinical and pre-clinical data.

The workshop participants also emphasized the importance of furthering communication, coordination, and collaboration between basic scientists, clinicians, and bioengineers, as well as between academia, industry, and government.

\section{Acknowledgments}

\section{Participant list}

Beth Ansel, ${ }^{*}:$ Clinton Baird, Hulya Bayir, Gretchen Brophy, Ross Bullock, Tracy Chen, William Clarke, David Clifford, Guy Clifton, ${ }^{*}$ Robin Conwit, ${ }^{*}+$ William Coplin, Ken Curley, $\$$ Pramod Dash, Donald Denson, Rebecca Desrocher, Ramon Diaz-Arrastia, W. Dalton Dietrich, Billy Dunn,*: Emmeline Edwards, $\$$ Debra Egan, $\$$ Scott Emerson, Stephanie Fertig, ${ }^{*}$ Donald Fink, Candace Floyd, Natalie Getzoff, Paula 
Goforth, Edward D. Hall, Ramona Hicks, ${ }^{*}+$ David Hoyt, Scott Janis, ${ }^{*}$ Melissa Kaime, $\$$ Naomi Kleitman, $\$$ Steve Korn, $\$$ Walter Koroshetz, $*$ Michelle LaPlaca, Daniel Laskowitz, Henry Lew, $\$$ Jonathan Lifschitz, Geoffrey Ling, $\$$ Susan Margulies, * David Meaney, David Moore, $\$$ Barclay Morrison, Claudia Moy, Linda Noble, John Povlishock, Ramesh Raghupathi, Claudia Robertson, Courtney Robertson, Peter Rumm, Leslie Satin, Holly Soares, George Sopko, $\$$ James Stables, Pat Sullivan, Frank Tortella, + Robert Vandre, $+\mathrm{Mi}-$ chael Whalen, Michael Weinrich, $*$ David Wright, and Ross Zafonte. ( ${ }^{*}$ Member of the organizing committee.t Support for the workshop came from the National Institute of Neurological Disorders and Stroke, the National Institute of Child Health and Development, the National Institute Heart, Lung, and Blood Institute, and the Department of Veterans Affairs. The views expressed by the authors in this manuscript represent the professional opinions of the authors and are not an official document, guidance, or policy of the United States Government, the Department of Veterans Affairs, the Department of Defense, the Department of Health and Human Services, the National Institutes of Health, or the U.S. Food and Drug Administration, nor should any official endorsement be inferred. Specific recommendations made in this manuscript should not be construed in any way to represent official recommendations or opinions of the FDA).

\section{Author Disclosure Statement}

No conflicting financial interests exist.

\section{References}

Adams, J.H., Graham, D.I., and Gennarelli, T.A. (1983). Head injury in man and experimental animals: neuropathology. Acta Neurochir. Suppl. (Wien.) 32, 15-30.

Adams, J.H., Graham, D.I., Murray, L.S., and Scott, G. (1982). Diffuse axonal injury due to nonmissile head injury in humans: an analysis of 45 cases. Ann. Neurol, 12, 557-563.

Adibhatla, R.M., and Hatcher, J.F. (2002). Citicoline mechanisms and clinical efficacy in cerebral ischemia. J. Neurosci. Res. 70, 133-139.

Adibhatla, R.M., and Hatcher, J.F. (2005). Cytidine 5'diphosphocholine (CDP-choline) in stroke and other CNS disorders. Neurochem. Res. 30, 15-23.

Ahmed, S.M., Rzigalinski, B.A., Willoughby, K.A., Sitterding, H.A., and Ellis, E.F. (2000). Stretch-induced injury alters mitochondrial membrane potential and cellular ATP in cultured astrocytes and neurons. J. Neurochem. 74, 1951-1960.

Alessandri, B., Rice, A.C., Levasseur, J., DeFord, M., Hamm, R.J., and Bullock, M.R. (2002). Cyclosporin A improves brain tissue oxygen consumption and learning/memory performance after lateral fluid percussion injury in rats. J. Neurotrauma 19, 829-841.

Allen, J.W., Knoblach, S.M., and Faden, A.I. (1999). Combined mechanical trauma and metabolic impairment in vitro induces NMDA receptor-dependent neuronal cell death and caspase3-dependent apoptosis. FASEB J. 13, 1875-1882.

Aronowski, J., Strong, R., Shirzadi, A., and Grotta, J.C. (2003). Ethanol plus caffeine (caffeinol) for treatment of ischemic stroke: preclinical experience. Stroke 34, 1246-1251.

Arundine, M., Aarts, M., Lau, A., and Tymianski, M. (2004). Vulnerability of central neurons to secondary insults after in vitro mechanical stretch. J. Neurosci. 24, 8106-8123.
Atkins, C.M., Oliva, A.A., Jr., Alonso, O.F., Chen, S., Bramlett, H.M., Hu, B.R., and Dietrich, W.D. (2007). Hypothermia treatment potentiates ERK1/2 activation after traumatic brain injury. Eur. J. Neurosci. 26, 810-819.

Bahr, B.A., Kessler, M., Rivera, S., Vanderklish, P.W., Hall, R.A., Mutneja, M.S., Gall, C., and Hoffman, K.B. (1995). Stable maintenance of glutamate receptors and other synaptic components in long-term hippocampal slices. Hippocampus 5, 425-439.

Baranova, A., Wei, E., Ueda, Y., Sholley, M., Kontos, H., and Povlishock, J. (2008). Cerebral vascular responsiveness after experimental traumatic brain injury: the beneficial effects of delayed hypothermia combined with superoxide dismutase administration. J. Neurosurg. 109, 502-509.

Baulieu, E.E., and Schumacher, M. (1997). Neurosteroids, with special reference to the effect of progesterone on myelination in peripheral nerves. Mult. Scler. 3, 105-112.

Bendel, O., Langmoen, I.A., and von Euler, G. (2004). Crush injury induces NMDA-receptor-dependent delayed nerve cell death in rat entorhinal-hippocampal slice cultures. Brain Res. $1025,35-42$.

Brain Trauma Foundation. (2007). Guidelines for the management of severe head injury. J. Neurotrauma 24(Suppl. 1), S1S106.

Bramlett, H.M., Dietrich, W.D., Green, E.J., and Busto, R. (1997). Chronic histopathological consequences of fluid-percussion brain injury in rats: effects of post-traumatic hypothermia. Acta Neuropathol. 93, 190-199.

Brines, M.L., Ghezzi, P., Keenan, S., Agnello, D., de Lanerolle, N.C., Cerami, C., Itri. L.M., and Cerami, A. (2000). Erythropoietin crosses the blood-brain barrier to protect against experimental brain injury. Proc. Natl. Acad. Sci. USA 97, 10526-10531.

Buchs, P.A., Stoppini, L., and Muller, D. (1993). Structural modifications associated with synaptic development in area CA1 of rat hippocampal organotypic cultures. Brain Res. Dev. Brain Res. 71, 81-91.

Caeser, M., and Aertsen, A. (1991). Morphological organization of rat hippocampal slice cultures. J. Comp. Neurol. 307, 87106.

Camacho-Arroyo, I., Pasapera, A.M., and Cerbon, M.A. (1996). Regulation of progesterone receptor gene expression by sex steroid hormones in the hypothalamus and the cerebral cortex of the rabbit. Neurosci. Lett. 214, 25-28.

Camacho-Arroyo, I., Perez-Palacios, G., Pasapera, A.M., and Cerbon, M.A. (1994). Intracellular progesterone receptors are differentially regulated by sex steroid hormones in the hypothalamus and the cerebral cortex of the rabbit. J. Steroid Biochem. Mol. Biol. 50, 299-303.

Cargill, R.S., 2nd, and Thibault, L.E. (1996). Acute alterations in $[\mathrm{Ca} 2+] \mathrm{i}$ in NG108-15 cells subjected to high strain rate deformation and chemical hypoxia: an in vitro model for neural trauma. J. Neurotrauma 13, 395-407.

Centers for Disease Control and Prevention. (2000). Vital statistics. From http://www.cdc.gov/nchs/fastats/stroke.htm.

Chen, J., Zhang, Z.G., Li, Y., Wang, Y., Wang, L., Jiang, H., Zhang, C., Lu, M., Katakowski, M., Feldkamp, C.S., and Chopp, M. (2003). Statins induce angiogenesis, neurogenesis, and synaptogenesis after stroke. Ann. Neurol. 53, 743-751.

Chen, S.F., Hung, T.H., Chen, C.C., Lin, K.H., Huang, Y.N., Tsai, H.C., and Wang, J.Y. (2007). Lovastatin improves histological and functional outcomes and reduces inflammation after experimental traumatic brain injury. Life Sci. 81, 288-298.

Cherian, L., Goodman, J.C., and Robertson, C. (2007). Neuroprotection with erythropoietin administration following con- 
trolled cortical impact injury in rats. J. Pharmacol. Exp. Ther. 322, 789-794.

Chong, Z.Z., Kang, J.Q., and Maiese, K. (2003a). Erythropoietin fosters both intrinsic and extrinsic neuronal protection through modulation of microglia, Akt1, Bad, and caspasemediated pathways. Br. J. Pharmacol. 138, 1107-1118.

Chong, Z.Z., Lin, S.H., Kang, J.Q., and Maiese, K. (2003b). Erythropoietin prevents early and late neuronal demise through modulation of Akt1 and induction of caspase 1, 3, and 8 . J. Neurosci. Res. 71, 659-669.

Clark, R.S., Carcillo, J.A., Kochanek, P.M., Obrist, W.D., Jackson, E.K., Mi, Z., Wisneiwski, S.R., Bell, M.J., and Marion, D.W. (1997). Cerebrospinal fluid adenosine concentration and uncoupling of cerebral blood flow and oxidative metabolism after severe head injury in humans. Neurosurgery 41, 12841292; discussion 1292-1293.

Clifton, G.L., Jiang, J.Y., Lyeth, B.G., Jenkins, L.W., Hamm, R.J., and Hayes, R.L. (1991). Marked protection by moderate hypothermia after experimental traumatic brain injury. J. Cereb. Blood Flow Metab. 11, 114-121.

Cochrane. (2005). The Cochrane Collaboration Brain Injury Review. Brain Injury, Cochrane Collaboration.

Cohadon, F., Richer, E., and Poletto, B. (1982). [A precursor of phospholipids in the treatment of severe traumatic comas]. Neurochirurgie 28, 287-290.

Collin, C., Miyaguchi, K., and Segal, M. (1997). Dendritic spine density and LTP induction in cultured hippocampal slices. J. Neurophysiol 77, 1614-1623.

Corwin, H.L., Gettinger, A., Fabian, T.C., May, A., Pearl, R.G., Heard, S., An, R., Bowers, P.J., Burton, P., Klausner, M.A., and Corwin, M.J. (2007). Efficacy and safety of epoetin alfa in critically ill patients. N. Engl. J. Med. 357, 965-976.

Corwin, H.L., Gettinger, A., Pearl, R.G., Fink, M.P., Levy, M.M., Shapiro, M.J., Corwin, M.J., and Colton, T. (2002). Efficacy of recombinant human erythropoietin in critically ill patients: a randomized controlled trial. JAMA 288, 2827-2835.

Corwin, H.L., Gettinger, A., Rodriguez, R.M., Pearl, R.G., Gubler, K.D., Enny, C., Colton, T., and Corwin, M.J. (1999). Efficacy of recombinant human erythropoietin in the critically ill patient: a randomized, double-blind, placebo-controlled trial. Crit. Care Med. 27, 2346-2350.

Cotelle, P. (2006). Patented HIV-1 integrase inhibitors (19982005). Recent Patents Anti-Infect. Drug Disc. 1, 1-15.

Cucchiara, B., and Kasner, S.E. (2001). Use of statins in CNS disorders. J. Neurol. Sci. 187, 81-89.

De Simoni, A., Griesinger, C.B., and Edwards, F.A. (2003). Development of rat CA1 neurones in acute versus organotypic slices: role of experience in synaptic morphology and activity. J. Physiol. 550, 135-147.

Digicaylioglu, M., Bichet, S., Marti, H.H., Wenger, R.H., Rivas, L.A., Bauer, C., and Gassmann, M. (1995). Localization of specific erythropoietin binding sites in defined areas of the mouse brain. Proc. Natl. Acad. Sci. USA 92, 3717-3120.

Doppenberg, E.M., Choi, S.C., and Bullock, R. (2004). Clinical trials in traumatic brain injury: lessons for the future. J. Neurosurg. Anesthesiol. 16, 87-94.

Doyle, J.A., Davis, D.P., and Hoyt, D.B. (2001). The use of hypertonic saline in the treatment of traumatic brain injury. J. Trauma 50, 367-383.

Edwards, M.E., Wang, S.S., and Good, T.A. (2001). Role of viscoelastic properties of differentiated SH-SY5Y human neuroblastoma cells in cyclic shear stress injury. Biotechnol. Prog. 17, 760-767.

Elkin, B.S., and Morrison, B., 3rd (2007). Region-specific tolerance criteria for the living brain. Stapp Car Crash J. 51, 127-138.
Emery, D.G., Lucas, J.H., and Gross, G.W. (1987). The sequence of ultrastructural changes in cultured neurons after dendrite transection. Exp. Brain Res. 67, 41-51.

Engel, D.C., Slemmer, J.E., Vlug, A.S., Maas, A.I., and Weber, J.T. (2005). Combined effects of mechanical and ischemic injury to cortical cells: secondary ischemia increases damage and decreases effects of neuroprotective agents. Neuropharmacology 49, 985-995.

Etzion, Y., and Grossman, Y. (2000). Pressure-induced depression of synaptic transmission in the cerebellar parallel fibre synapse involves suppression of presynaptic N-type Ca2+ channels. Eur. J. Neurosci. 12, 4007-4016.

Faden, A.I. (2002). Neuroprotection and traumatic brain injury: theoretical option or realistic proposition. Curr. Opin. Neurol. $15,707-712$.

Ferrand-Drake, M., Zhu, C., Gido, G., Hansen, A.J., Karlsson, J.O., Bahr, B.A., Zamzami, N., Kroemer, G., Chan, P.H., Wieloch, T., and Blomgren, K. (2003). Cyclosporin A prevents calpain activation despite increased intracellular calcium concentrations, as well as translocation of apoptosis-inducing factor, cytochrome $\mathrm{c}$ and caspase- 3 activation in neurons exposed to transient hypoglycemia. J. Neurochem. 85, 1431-1442.

Feuerstein, G.Z., Zaleska, M.M., Krams, M., Wang, X., Day, M., Rutkowski, J.L., Finklestein, S.P., Pangalos, M.N., Poole, M., Stiles, G.L., Ruffolo, R.R., and Walsh, F.L. (2008). Missing steps in the STAIR case: a Translational Medicine perspective on the development of NXY-059 for treatment of acute ischemic stroke. J. Cereb. Blood Flow Metab. 28, 217-219.

Fisher, M., Feurstein, G., Howells, D.W., Hurn, P.D., Kent, T.A., Savitz, S.I., Lo, E.H. (2009). Update on stroke theraphy academic industry roundtable preclinical recommendations. Stroke DOI: 10.1161/STROKEAHA.108.541128.

Floyd, C.L., and Lyeth, B.G. (2007). Astroglia: important mediators of traumatic brain injury. Prog. Brain Res. 161, 61-79.

Floyd, R.A. (1999). Antioxidants, oxidative stress, and degenerative neurological disorders. Proc. Soc. Exp. Biol. Med. 222, 236-245.

Foda, M.A., and Marmarou, A. (1994). A new model of diffuse brain injury in rats. Part II: Morphological characterization. J. Neurosurg. 80, 301-313.

Folbergrova, J., Li, P.A., Uchino, H., Smith, M.L., and Siesjo, B.K. (1997). Changes in the bioenergetic state of rat hippocampus during $2.5 \mathrm{~min}$ of ischemia, and prevention of cell damage by cyclosporin A in hyperglycemic subjects. Exp. Brain Res. 114, 44-50.

Frantseva, M.V., Kokarovtseva, L., Naus, C.G., Carlen, P.L., MacFabe, D., and Perez, J.L. Velazquez, J.L. (2002). Specific gap junctions enhance the neuronal vulnerability to brain traumatic injury. J. Neurosci. 22, 644-653.

Fukui, S., Signoretti, A., Dunbar, J.G., and Marmarou, A. (2003). The effect of cyclosporin A on brain edema formation following experimental cortical contusion. Acta Neurochir. Suppl. 86, 301-303.

Gahwiler, B.H. (1984). Development of the hippocampus in vitro: cell types, synapses and receptors. Neuroscience 11, 751-760.

Genazzani, A.R., Petraglia, F., and Purdy, R.H. (1996). The brain: source and target for sex steroid hormones. 1st Tuscania Conference on Reproductive Medicine, Pisa, Italy. Parthenon Pub. Group: New York.

Glass, T.F., Reeves, B., and Sharp, F.R. (2002). Modeling both the mechanical and hypoxic features of traumatic brain injury in vitro in rats. Neurosci. Lett. 328, 133-136.

Glass, T.F., Reeves, B. and Sharp, F.R. (2004). The impact of excitotoxic blockade on the evolution of injury following 
combined mechanical and hypoxic insults in primary rat neuronal culture. Neurobiol. Dis. 17, 378-384.

Gorio, A., Madaschi, L., Di Stefano, B., Carelli, S., Di Giulio, A.M., De Biasi, S., Coleman, T., Cerami, A., and Brines, M. (2005). Methylprednisolone neutralizes the beneficial effects of erythropoietin in experimental spinal cord injury. Proc. Natl. Acad. Sci. USA 102, 16379-16384.

Graham, D.I., Gennarelli, T., and McIntosh, T.K. (2002). Trauma. Greenfields's Neuropathology. D.I. Graham and P.L. Lantos (eds), Arnold: New York, pp. 823-882.

Graham, D.I., McIntosh, T.K., Maxwell, W.L., and Nicoll, J.A. (2000). Recent advances in neurotrauma. J. Neuropathol. Exp. Neurol. 59, 641-651.

Grasso, G. (2001). Neuroprotective effect of recombinant human erythropoietin in experimental subarachnoid hemorrhage. J. Neurosurg. Sci. 45, 7-14.

Gross, G.W., and Higgins, M.L. (1987). Cytoplasmic damage gradients in dendrites after transection lesions. Exp. Brain Res. $67,52-60$.

Gross, G.W., Lucas, J.H., and Higgins, M.L. (1983). Laser microbeam surgery: ultrastructural changes associated with neurite transection in culture. J. Neurosci. 3, 1979-1993.

Hall, E.D., McCall, J.M., and Means, E.D. (1994). Therapeutic potential of the lazaroids (21-aminosteroids) in acute central nervous system trauma, ischemia and subarachnoid hemorrhage. Adv. Pharmacol. 28, 221-268.

Hansson, M.J., Persson, T., Friberg, H., Keep, M.F., Rees, A., Wieloch, T., and Elmer, E. (2003). Powerful cyclosporin inhibition of calcium-induced permeability transition in brain mitochondria. Brain Res. 960, 99-111.

Harrington, M., and Carpenter, C.C. (2000). Hit HIV-1 hard, but only when necessary. Lancet 355, 2147-2152.

Hartel, R., and Matus, A. (1997). Cytoskeletal maturation in cultured hippocampal slices. Neuroscience 78, 1-5.

Hickenbottom, S.L., and Grotta, J. (1998). Neuroprotective therapy. Semin. Neurol. 18, 485-492.

Holtgrave, D.R. (2005). Causes of the decline in AIDS deaths, United States, 1995-2002: prevention, treatment or both? Int. J. STD AIDS 16, 777-781.

Hong, S.J., and Chang, C.C. (1995). Inhibition of acetylcholine release from mouse motor nerve by a P-type calcium channel blocker, omega-agatoxin IVA. J. Physiol. 482, 283-290.

Jung, K.H., Chu, K., Jeong, S.W., Han, S.y., Lee, S.T., Kim, J.Y., Kim, M., and Roh, J.K. (2004). HMG-CoA reductase inhibitor, atorvastatin, promotes sensorimotor recovery, suppressing acute inflammatory reaction after experimental intracerebral hemorrhage. Stroke 35, 1744-1749.

Juul, S.E., Stallings, S.A., and Christensen, R.D. (1999). Erythropoietin in the cerebrospinal fluid of neonates who sustained CNS injury. Pediatr. Res. 46, 543-547.

Kaibara, T., Sutherland, G.R., Colbourne, F., and Tyson, R.L. (1999). Hypothermia: depression of tricarboxylic acid cycle flux and evidence for pentose phosphate shunt upregulation. J. Neurosurg. 90, 339-347.

Kamada, M., Li, R.Y., Hashimoto, M., Kakuda, M., Okada, H., Koyanagi, Y., Ishizuka, T., and Yawo, H. (2004). Intrinsic and spontaneous neurogenesis in the postnatal slice culture of rat hippocampus. Eur. J. Neurosci. 20, 2499-2508.

Kanagy, N.L., Perrine, M.F., Cheung, D.K., and Walker, B.R. (2003). Erythropoietin administration in vivo increases vascular nitric oxide synthase expression. J. Cardiovasc. Pharmacol. 42, 527-533.

Katano, H., Fujita, K., Kato, T., Asai, K., Kawamura, Y., Masago, A., and Yamada, K. (1999). Traumatic injury in vitro induces
IEG mRNAs in cultured glial cells, suppressed by co-culture with neurons. Neuroreport 10, 2439-2348.

Kennedy, J.E., Clement, P.F., and Curtiss, G. (2003). WAIS-III processing speed index scores after TBI: the influence of working memory, psychomotor speed and perceptual processing. Clin. Neuropsychol. 17, 303-307.

Koizumi, H., and Povlishock, J.T. (1998). Posttraumatic hypothermia in the treatment of axonal damage in an animal model of traumatic axonal injury. J. Neurosurg. 89, 303-309.

LaPlaca, M.C., and Thibault, L.E. (1997). An in vitro traumatic injury model to examine the response of neurons to a hydrodynamically-induced deformation. Ann. Biomed. Eng. 25, 665-677.

Laufs, U., La Fata, V., Plutzky, J., and Liao, J.K. (1998). Upregulation of endothelial nitric oxide synthase by HMG CoA reductase inhibitors. Circulation 97, 1129-1135.

Lea, P.M.T., Custer, S.J., Stoica, B.A., and Faden, A.I. (2003). Modulation of stretch-induced enhancement of neuronal NMDA receptor current by mGluR1 depends upon presence of glia. J. Neurotrauma 20, 1233-1249.

Lea, P.M., Custer, S.J., Vicini, S., and Faden, A.I. (2002). Neuronal and glial mGluR5 modulation prevents stretch-induced enhancement of NMDA receptor current. Pharmacol. Biochem. Behav. 73, 287-298.

Lefkopoulou, M., and Ryan, L. (1993). Global tests for multiple binary outcomes. Biometrics 49, 975-988.

Levin, H.S. (1991). Treatment of postconcussional symptoms with CDP-choline. J. Neurol. Sci. 103(Suppl.), S39-S42.

Limmroth, V., Lee, W.S., and Moskowitz, M.A. (1996). GABAAreceptor-mediated effects of progesterone, its ring-A-reduced metabolites and synthetic neuroactive steroids on neurogenic oedema in the rat meninges. Br. J. Pharmacol. 117, 99-104.

Li, P.A., Kristian, T., He, Q.P., and Siesjo, B.K. (2000). Cyclosporin A enhances survival, ameliorates brain damage, and prevents secondary mitochondrial dysfunction after a 30-minute period of transient cerebral ischemia. Exp. Neurol. 165, 153-163.

Lo, E.H., Wang, X., and Cuzner, M.L. (2002). Extracellular proteolysis in brain injury and inflammation: role for plasminogen activators and matrix metalloproteinases. J. Neurosci. Res. 69, 1-9.

Lu, M., and Tilley, B.C. (2001). Use of odds ratio or relative risk to measure a treatment effect in clinical trials with multiple correlated binary outcomes: data from the NINDS t-PA stroke trial. Stat. Med. 20, 1891-1901.

Lu, D., Goussev, A., Chen, J., Pannu, P., Li, Y., Mahmood, A., and Chopp, M. (2004a). Atorvastatin reduces neurological deficit and increases synaptogenesis, angiogenesis, and neuronal survival in rats subjected to traumatic brain injury. J. Neurotrauma 21, 21-32.

Lu, D., Mahmood, A., Goussev, A., Schallert, T., Qu, C., Zhang, Z.G., Li, Y., Lu, M., and Chopp, M. (2004b). Atorvastatin reduction of intravascular thrombosis, increase in cerebral microvascular patency and integrity, and enhancement of spatial learning in rats subjected to traumatic brain injury. J. Neurosurg. 101, 813-821.

Lu, D., Mahmood, A., Qu, C., Goussev, A., Lu, M., and Chopp, M. (2004c). Atorvastatin reduction of intracranial hematoma volume in rats subjected to controlled cortical impact. J. Neurosurg. 101, 822-825.

Lu, D., Mahmood, A., Qu, C., Goussev, A., Schallert, T., and Chopp, M. (2005). Erythropoietin enhances neurogenesis and restores spatial memory in rats after traumatic brain injury. J. Neurotrauma 22, 1011-1017.

Lu, D., Qu, C., Goussev, A., Jiang, H., Lu, C., Schallert, T., Mahmood, A., Chen, J., Li, Y., and Chopp, M. (2007). Statins 
increase neurogenesis in the dentate gyrus, reduce delayed neuronal death in the hippocampal CA3 region, and improve spatial learning in rat after traumatic brain injury. J. Neurotrauma 24, 1132-1146.

Lucas, J.H., Emery, D.G., Higgins, M.L., and Gross, G.W. (1990). Neuronal survival and dynamics of ultrastructural damage after dendrotomy in low calcium. J. Neurotrauma 7, 169-192.

Lynch, J.R., Wang, H., McGirt, M.J., Floyd, J., Friedman, A.H., Coon, A.L., Blessing, R., Alexander, M.J., Graffagnino, C., Warner, D.S., and Laskowitz, D.T. (2005). Simvastatin reduces vasospasm after aneurysmal subarachnoid hemorrhage: results of a pilot randomized clinical trial. Stroke 36, 2024-2026.

Maas, A.I., Marmarou, A., Murray, G.D., Teasdale, S.G., and Steyerberg, E.W. (2007). Prognosis and clinical trial design in traumatic brain injury: the Impact study. J. Neurotrauma 24, 232-238.

Maas, A.I. (2001). Neuroprotective agents in traumatic brain injury. Expert Opin. Investig. Drugs 10, 753-767.

Mansfield, R.T., Schiding, J.K., Hamilton, R.L., and Kochanek, P.M. (1996). Effects of hypothermia on traumatic brain injury in immature rats. J. Cereb. Blood Flow Metab. 16, 244-252.

Marklund, N., Bakshi, A., Castelbuono, D.J., Conte, V., and McIntosh, T.K. (2006). Evaluation of pharmacological treatment strategies in traumatic brain injury. Curr. Pharm. Des. $12,1645-1680$.

Marti, H.H., Bernaudin, M., Petit, E., and Bauer, C. (2000). Neuroprotection and Angiogenesis: Dual role of erythropoietin in brain ischemia. News Physiol. Sci. 15, 225-229.

May, M.T., Sterne, J.A., Costagliola, D., Sabin, C.A., Phillips, A.N., Justice, A.C., Dabis, F., Gill, J., Lundgren, J., Hogg, R.S., de Wolf, F., Fatkenheuer, G., Staszewski, S., d'Arminio Monforte, A., and Egger, M. (2006). HIV treatment response and prognosis in Europe and North America in the first decade of highly active antiretroviral therapy: a collaborative analysis. Lancet 368, 451-458.

Mbye, L.H., Singh, I.N., Carrico, K.M., Saatman, K.E., and Hall, E.D. (2009). Comparative neuroprotective effects of cyclosporin A and NIM811, a nonimmunosuppressive cyclosporin A analog, following traumatic brain injury. J. Cereb. Blood Flow Metab. 29, 87-97.

McCauley, S.R., Levin, H.S., Vanier, M., Mazaux, J.M., Boake, C., Goldfader, P.R., Rockers, D., Butters, M., Kareken, D.A., Lambert, J., and Clifton, G.L. (2001). The neurobehavioural rating scale-revised: sensitivity and validity in closed head injury assessment. J. Neurol. Neurosurg. Psychiatry 71, 643-651.

McGirt, M.J., Lynch, J.R., Parra, A., Sheng, H., Pearlstein, R.D., Laskowitz, D.T., Pelligrino, D.A., and Warner, D.S. (2002). Simvastatin increases endothelial nitric oxide synthase and ameliorates cerebral vasospasm resulting from subarachnoid hemorrhage. Stroke 33, 2950-2956.

McIntosh, T.K., Smith, D.H., Meaney, D.F., Kotapka, M.J., Gennarelli, T.A., and Graham, D.I. (1996). Neuropathological sequelae of traumatic brain injury: relationship to neurochemical and biomechanical mechanisms. Lab. Invest. 74, 315-342.

Mitsuyasu, R. (2002). Immune therapy: non-highly active antiretroviral therapy management of human immunodeficiency virus-infected patients. J. Infect. Dis. 185(Suppl. 2), S115-S122.

Moppett, I.K. (2007). Traumatic brain injury: assessment, resuscitation and early management. Br. J. Anaesth. 99, 18-31.

Morganti-Kossmann, M.C., Satgunaseelan, L., Bye, N., and Kossmann, T. (2007). Modulation of immune response by head injury. Injury 38, 1392-1400.

Morrison, B., 3rd, Cater, H.L., Wang, C.C., Thomas, F.C., Hung, C.T., Ateshian, G.A., and Sundstrom, L.E. (2003). A tissue level tolerance criterion for living brain developed with an in vitro model of traumatic mechanical loading. Stapp Car Crash J. 47, 93-105.

Mukhin, A.G., Ivanova, S.A., Allen, J.W., and Faden, A.I. (1998). Mechanical injury to neuronal/glial cultures in microplates: role of NMDA receptors and $\mathrm{pH}$ in secondary neuronal cell death. J. Neurosci. Res. 51, 748-758.

Mukhin, A.G., Ivanova, S.A., Knoblach, S.M., and Faden, A.I. (1997). New in vitro model of traumatic neuronal injury: evaluation of secondary injury and glutamate receptormediated neurotoxicity. J. Neurotrauma 14, 651-663.

Narayan, R.K., Michel, M.E., Ansell, B., Baethmann, A., Biegon, A., Bracken, M.B., Bullock, M.R., Choi, S.C., Clifton, G.L., Contant, C.F., Coplin, W.M., Dietrich, W.D., Ghajar, J., Grady, S.M., Grossman, R.G., Hall, E.D., Heetderks, W., Hovda, D.A., Jallo, J., Katz, R.L., Knoller, N., Kochanek, P.M., Maas, A.I., Majde, J., Marion, D.W., Marmarou, A., Marshall, L.F., McIntosh, T.K., Miller, E., Mohberg, N., Muizelaar, J.P., Pitts, L.H., Quinn, P., Riesenfeld, G., Robertson, C.S., Strauss, K.I., Teasdale, G., Temkin, N., Tuma, R., Wade, C., Walker, M.D., Weinrich, M., Whyte, J., Wilberger, J., Young, A.B., and Yurkewicz, L. (2002). Clinical trials in head injury. J. Neurotrauma 19, 503-557.

Palesch, Y.Y., Tilley, B.C., Sackett, D.L., Johnston, K.C., and Woolson, R. (2005). Applying a phase II futility study design to therapeutic stroke trials. Stroke 36, 2410-2414.

Panickar, K.S., Jayakumar, A.R., and Norenberg, M.D. (2002). Differential response of neural cells to trauma-induced free radical production in vitro. Neurochem. Res. 27, 161-166.

Panizzon, K.L., Shin, D., Frautschy, S., and Wallis, R.A. (1998). Neuroprotection with Bcl-2(20-34) peptide against trauma. Neuroreport 9, 4131-4136.

Pascual, J.L., Khwaja, K.A., Ferri, L.E., Giannias, B., Evans, D.C., Razek, T., Michel, R.P., and Christou, N.V. (2003). Hypertonic saline resuscitation attenuates neutrophil lung sequestration and transmigration by diminishing leukocyte-endothelial interactions in a two-hit model of hemorrhagic shock and infection. J. Trauma 54, 121-130; discussion 130-132.

Pasternak, J.J., and Lanier, W.L. (2007). Neuroanesthesiology review-2006. J. Neurosurg. Anesthesiol. 19, 70-92.

Peterson, K., Carson, S., and Carney, N. (2008). Hypothermia treatment for traumatic brain injury: a systematic review and meta-analysis. J. Neurotrauma 25, 62-71.

Povlishock, J.T., Becker, D.P., Cheng, C.L., and Vaughan, G.W. (1983). Axonal change in minor head injury. J. Neuropathol. Exp. Neurol. 42, 225-242.

Povlishock, J.T., and Katz, D.I. (2005). Update of neuropathology and neurological recovery after traumatic brain injury. J. Head Trauma Rehabil. 20, 76-94.

Povlishock, J.T. (1992). Traumatically induced axonal injury: pathogenesis and pathobiological implications. Brain Pathol. 2, 1-12.

Prough, D.S., Kramer, G.C., Uchida, T., Stephenson, R.T., Hellmich, H.L., and Dewitt, D.S. (2006). Effects of hypertonic arginine on cerebral blood flow and intracranial pressure after traumatic brain injury combined with hemorrhagic hypotension. Shock 26, 290-295.

Raghupathi, R. (2004). Cell death mechanisms following traumatic brain injury. Brain Pathol. 14, 215-222.

Reddy, D.S., and Kulkarni, S.K. (1996). Role of GABA-A and mitochondrial diazepam binding inhibitor receptors in the anti-stress activity of neurosteroids in mice. Psychopharmacology (Berl.) 128, 280-292.

Robain, O., Barbin, G., Billette de Villemeur, T., Jardin, L., Jahchan, T., and Ben-Ari, Y. (1994). Development of mossy fiber 
synapses in hippocampal slice culture. Brain Res. Dev. Brain Res. 80, 244-250.

Roberts, I., Schierhout, G., and Alderson, P. (1998). Absence of evidence for the effectiveness of five interventions routinely used in the intensive care management of severe head injury: a systematic review [see comments]. J. Neurol. Neurosurg. Psychiatry 65, 729-733.

Saatman, K.E., Duhaime, A.-C., Bullock, R., Maas, A.I.R., Valadka, A., Manley, G.T., and Workshop Scientific Team and Advisory Panel Members (2008). Classification of traumatic brain injury for targeted therapies. J. Neurotrauma 25, 719738.

Sahuquillo, J., and Vilalta, A. (2007). Cooling the injured brain: how does moderate hypothermia influence the pathophysiology of traumatic brain injury. Curr. Pharm. Des. 13, 23102322.

Salzwedel, K., Martin, D.E., and Sakalian, M. (2007). Maturation inhibitors: a new therapeutic class targets the virus structure. AIDS Rev. 9, 162-172.

Schouten, J.W. (2007). Neuroprotection in traumatic brain injury: a complex struggle against the biology of nature. Curr. Opin. Crit. Care 13, 134-142.

Schwid, S.R., and Cutter, G.R. (2006). Futility studies: spending a little to save a lot. Neurology 66, 626-627.

Secades, J.J., and Lorenzo, J.L. (2006). Citicoline: pharmacological and clinical review, 2006 update. Methods Find Exp. Clin. Pharmacol. 28(Suppl. B), 1-56.

Serbest, G., Horwitz, J., and Barbee, K. (2005). The effect of poloxamer-188 on neuronal cell recovery from mechanical injury. J. Neurotrauma 22, 119-132.

Seyfried, D., Han, Y., Lu, D., Chen, J., Bydon, A., and Chopp, M. (2004). Improvement in neurological outcome after administration of atorvastatin following experimental intracerebral hemorrhage in rats. J. Neurosurg. 101, 104-107.

Sieg, F., Wahle, P., and Pape, H.C. (1999). Cellular reactivity to mechanical axonal injury in an organotypic in vitro model of neurotrauma. J. Neurotrauma 16, 1197-1213.

Signoretti, S., Marmarou, A., Tavazzi, B., Dunbar, J., Amorini, A.M., Lazzarino, G., and Vagnozzi, R. (2004). The protective effect of cyclosporin A upon $\mathrm{N}$-acetylaspartate and mitochondrial dysfunction following experimental diffuse traumatic brain injury. J. Neurotrauma 21, 1154-1167.

Smith, S.L., and Hall, E.D. (1996). Mild pre- and posttraumatic hypothermia attenuates blood-brain barrier damage following controlled cortical impact injury in the rat. J. Neurotrauma 13, $1-9$.

Smith, D.H., Meaney, D.F., and Shull, W.H. (2003). Diffuse axonal injury in head trauma. J. Head Trauma Rehabil. 18, 307316.

Smith, D.H., Wolf, J.A., Lusardi, T.A., Lee, V.M., and Meaney, D.F. (1999). High tolerance and delayed elastic response of cultured axons to dynamic stretch injury. J. Neurosci. 19, 4263-4269.

Sofroniew, M.V. (2005). Reactive astrocytes in neural repair and protection. Neuroscientist 11, 400-407.

Springborg, J.B., Ma, X., Rochat, P., Knudsen, G.M., Amtorp, O., Paulson, O.B., Juhler, M., and Olsen, N.V. (2002). A single subcutaneous bolus of erythropoietin normalizes cerebral blood flow autoregulation after subarachnoid haemorrhage in rats. Br. J. Pharmacol. 135, 823-829.

STAIR-II (Stroke Therapy Academic Industry Roundtable II). (2001). Recommendations for clinical trial evaluation of acute stroke therapies. Stroke 32, 1598-1606.

STAIR (Stroke Therapy Academic Industry Roundtable). (1999). Recommendations for standards regarding preclinical neuro- protective and restorative drug development. Stroke 30, 27522758.

Stein, D.G., Wright, D.W., and Kellermann, A.L. (2008). Does progesterone have neuroprotective properties? Ann. Emerg. Med. 51, 164-172.

Stoppini, L., Buchs, P.A., and Muller, D. (1993). Lesion-induced neurite sprouting and synapse formation in hippocampal organotypic cultures. Neuroscience 57, 985-994.

Strong, R., Grotta, J.C., and Aronowski, J. (2000). Combination of low dose ethanol and caffeine protects brain from damage produced by focal ischemia in rats. Neuropharmacology 39, 515-522.

Suehiro, E., Ueda, Y., Wei, E.P., Kontos, H.A., and Povlishock, J.T. (2003). Posttraumatic hypothermia followed by slow rewarming protects the cerebral microcirculation. J. Neurotrauma 20, 381-390.

Sullivan, P.G., Rabchevsky, A.G., Waldmeier, P.C., and Springer, J.E. (2005). Mitochondrial permeability transition in CNS trauma: cause or effect of neuronal cell death? J. Neurosci. Res. 79, 231-239.

Sullivan, P.G., Thompson, M., and Scheff, S.W. (2000). Continuous infusion of cyclosporin A postinjury significantly ameliorates cortical damage following traumatic brain injury. Exp. Neurol. 161, 631-637.

Tecoma, E.S., Monyer, H., Goldberg, M.P., and Choi, D.W. (1989). Traumatic neuronal injury in vitro is attenuated by NMDA antagonists. Neuron 2, 1541-1545.

Temkin, N.R., Anderson, G.D., Winn, H.R., Ellenbogen, R.G., Britz, G.W., Schuster, J., Lucas, T., Newell, D.W., Mansfield, P.N., Machamer, J.E., Barber, J., and Dikmen, S.S. (2007). Magnesium sulfate for neuroprotection after traumatic brain injury: a randomised controlled trial. Lancet Neurol. 6, 29-38.

Thompson, H.J., Lifshitz, J., Marklund, N., Grady, M.S., Graham, D.I., Hovda, D.A., and McIntosh, T.K. (2005). Lateral fluid percussion brain injury: a 15-year review and evaluation. J. Neurotrauma 22, 42-75.

Tilley, B.C., Palesch, Y.Y., Kieburtz, K., Ravina, B., Huang, P., Elm, J.J., Shannon, K., Wooten, G.F., Tanner, C.M., and Goetz, G.C. (2006). Optimizing the ongoing search for new treatments for Parkinson disease: using futility designs. Neurology 66, 628-633.

Tommasino, C., and Picozzi, V. (2007). Volume and electrolyte management. Best Pract. Res. Clin. Anaesthesiol. 21, 497-516.

Toni, N., Stoppini, L., and Muller, D. (1997). Staurosporine but not chelerythrine inhibits regeneration in hippocampal organotypic cultures. Synapse 27, 199-207.

Tortorici, M.A., Kochanek, P.M., and Poloyac, S.M. (2007). Effects of hypothermia on drug disposition, metabolism, and response: A focus of hypothermia-mediated alterations on the cytochrome P450 enzyme system. Crit. Care Med. 35, 21962204.

Tseng, M.Y., Czosnyka, M., Richards, H., Pickard, J.D., and Kirkpatrick, P.J. (2005). Effects of acute treatment with pravastatin on cerebral vasospasm, autoregulation, and delayed ischemic deficits after aneurysmal subarachnoid hemorrhage: a phase II randomized placebo-controlled trial. Stroke 36, $1627-1632$.

U.S. Food and Drug Administration. (2008). Retrieved 6/16/08, 2008, from http://www.accessdata.fda.gov/scripts/cdrh/cfdocs/ cfccfr/CFRSearch.cfm.

Unterberg, A.W., Stover, J., Kress, B., and Kiening, K.L. (2004) Edema and brain trauma. Neuroscience 129, 1021-1029.

Vink, R., McIntosh, T.K., Weiner, M.W., and Faden, A.I. (1987). Effects of traumatic brain injury on cerebral high-energy 
phosphates and $\mathrm{pH}$ : a 31P magnetic resonance spectroscopy study. J. Cereb. Blood Flow Metab. 7, 563-571.

Wang, H., Lynch, J.R., Song, P., Yang, H.J., Yates, R.B., Mace, B., Warner, D.S., Guyton, J.R., and Laskowitz, D.T. (2007). Simvastatin and atorvastatin improve behavioral outcome, reduce hippocampal degeneration, and improve cerebral blood flow after experimental traumatic brain injury. Exp. Neurol. 206, 59-69.

Wang, L., Zhang, Z., Wang, Y., Zhang, R., and Chopp, M. (2004). Treatment of stroke with erythropoietin enhances neurogenesis and angiogenesis and improves neurological function in rats. Stroke 35, 1732-1737.

Warach, S., Pettigrew, L.C., Dashe, J.F., Pullicino, P., Lefkowitz, D.M., Sabounjian, L., Harnett, K., Schwiderski, U., and Gammans, R. (2000). Effect of citicoline on ischemic lesions as measured by diffusion-weighted magnetic resonance imaging. Citicoline 010 Investigators. Ann. Neurol. 48, 713-722.

Weitz-Schmidt, G., Welzenbach, K., Brinkmann, V., Kamata, T., Kallen, J., Bruns, C., Cottens, S., Takada, Y., and Hommel, U. (2001). Statins selectively inhibit leukocyte function antigen- 1 by binding to a novel regulatory integrin site. Nat. Med. 7, 687-692.

Werner, C., and Engelhard, K. (2007). Pathophysiology of traumatic brain injury. Br. J. Anaesth. 99, 4-9.

Wright, D.W., Bauer, M.E., Hoffman, S.W., and Stein, D.G. (2001). Serum progesterone levels correlate with decreased cerebral edema after traumatic brain injury in male rats. J. Neurotrauma 18, 901-909.

Wright, D.W., Kellermann, A.L., Hertzberg, V.S., Clark, P.L., Frankel, M., Goldstein, F.C., Salomone, J.P., Dent, L.L., Harris, O.A., Ander, D.S., Lowery, D.W., Patel, M.M., Denson, D.D., Gordon, A.B., Wald, M.M., Gupta, S., Hoffman, S.W., and Stein, D.G. (2007). ProTECT: a randomized clinical trial of progesterone for acute traumatic brain injury. Ann. Emerg. Med. 49, 391-402, 402e1-2.

Xiao, G., Wei, J., Yan, W., Wang, W., and Lu, Z. (2008). Improved outcomes from the administration of progesterone for patients with acute severe traumatic brain injury: a randomized controlled trial. Crit. Care 12, R61.

Yeni, P.G., Hammer, S.M., Carpenter, C.C., Cooper, D.A., Fischl, M.A., Gatell, J.M., Gazzard, B.G., Hirsch, M.S., Jacobsen, D.M., Katzenstein, D.A., Montaner, J.S., Richman, D.D., Saag, M.S., Schechter, M., Schooley, R.T., Thompson, M.A., Vella, S., and Volberding, P.A. (2002). Antiretroviral treatment for adult HIV infection in 2002: updated recommendations of the International AIDS Society-USA Panel. JAMA 288, 222-235.

Yi, J.H., and Hazell, A.S. (2006). Excitotoxic mechanisms and the role of astrocytic glutamate transporters in traumatic brain injury. Neurochem. Int. 48, 394-403.

Zimmer, J., and Gahwiler, B.H. (1984). Cellular and connective organization of slice cultures of the rat hippocampus and fascia dentata. J. Comp. Neurol. 228, 432-446.

Address reprint requests to: Susan Margulies, Ph.D. Professor of Bioengineering School of Engineering and Applied Science Department of Bioengineering University of Pennsylvania 240 Skirkanich Hall 210 S. 33rd Street Philadelphia, PA 19104-6321 E-mail: margulie@seas.upenn.edu 
